\title{
Wallenda regulates JNK-mediated cell death in Drosophila
}

\author{
$X M^{*, 1,2}, W X u^{1}, D$ Zhang ${ }^{1}, Y$ Yang ${ }^{1}, W L^{1}$ and $L X u e^{*, 1}$
}

The c-Jun N-terminal kinase (JNK) pathway plays essential roles in regulating a variety of cellular processes including proliferation, migration and survival. Previous genetic studies in Drosophila have identified numerous cell death regulating genes, providing new insights into the mechanisms for related diseases. Despite the known role of the small GTPase Rac1 in regulating cell death, the downstream components and underlying mechanism remain largely elusive. Here, we show that Rac1 promotes JNKdependent cell death through Wallenda (Wnd). In addition, we find that Wnd triggers JNK activation and cell death via its kinase domain. Moreover, we show that both MKK4 and Hep are critical for Wnd-induced cell death. Furthermore, Wnd is essential for ectopic Egr- or Rho1-induced JNK activation and cell death. Finally, Wnd is physiologically required for loss of scribble-induced JNK-dependent cell death. Thus, our data suggest that wnd encodes a novel essential cell death regulator in Drosophila.

Cell Death and Disease (2015) 6, e1737; doi:10.1038/cddis.2015.111; published online 7 May 2015

Programmed cell death (PCD) is a fundamental biological process required for normal organ development and tissue homeostasis in multicellular organisms. ${ }^{1}$ Disruption of PCD would result in a variety of diseases including neurodegenerative diseases, autoimmune disorders and cancers. $^{2}$ Drosophila melanogaster, with its well-established genetic techniques and compact genome size, has been regarded as an excellent model organism to study PCD and its related signaling pathways. ${ }^{3,4}$ The c-Jun N-terminal kinase (JNK) signaling has been implicated as one of the most important pathways that regulates various fundamental cell behaviors, such as proliferation, migration and cell death. 5,6

Rac1 belongs to the Rho family of small GTPase that regulates many aspects of physiological activities ranging from immune response to wound healing and migration. ${ }^{7-11}$ For instance, Rac1 has been implicated in JNK-mediated dorsal closure via Slpr (Slipper) in fly, ${ }^{7}$ osteoclast differentiation through TAK1-mediated NF- $\kappa B$ signaling ${ }^{12}$ and myocyte hypertrophy via Ask1 (apoptotic signal-regulating kinase 1) in mammals. ${ }^{13}$ However, despite the reported role of Rac 1 in cell death, ${ }^{14}$ its underlying mechanism and downstream components remain largely elusive.

Here by using Drosophila compound eye as a model, we found Rac1 expression induces JNK-dependent cell death and identified Wallenda (Wnd), a MAPKKK (mitogen-activated protein kinase kinase kinase) member as an essential downstream mediator. Furthermore, we found that Wnd is sufficient to induce JNK-mediated cell death through both Hep and MKK4. Finally, we established Wnd as a general modulator of cell death in Drosophila by showing that it is also required for ectopic Egr or Rho1 and loss of Scribble (Scrib)-induced cell death.

\section{Results and Discussion}

Wnd is essential for Rac1-induced cell death and JNK activation. Consistent with previous results that overexpression of the small GTPase Rac1 would affect eye development, ${ }^{7,14}$ we found that expression of Rac1 under GMR promoter produced a complete eye loss phenotype (Figure $1 b$ ), resulting from extensive cell death posterior to the morphogenetic furrow (MF) in third instar eye discs (Figure 2f), as shown by acridine orange (AO) staining, a dye used to detect dying cells. ${ }^{15}$ In accordance with the genetic evidence that Rac1 regulates JNK-mediated dorsal closure, ${ }^{10}$ we found that blocking JNK activity by expressing a dominant negative allele of Bsk $\left(\mathrm{Bsk}^{\mathrm{DN}}\right)$ or the JNK phosphatase Puc could dramatically suppress Rac1-triggered eye loss phenotype (Figures 1c and d), although some pigment cells defects still remain. Furthermore, knocking down either of the two JNK kinases, Hemipterous (Hep) or MKK4, significantly suppressed Rac1-triggered no-eye phenotype (Figures $1 \mathrm{e}$ and $\mathrm{f}$ ), indicating a critical role of JNK signaling in Rac1-induced cell death.

In Drosophila, upstream of Hep and MKK4 in the JNK pathway are five JNKKKs, including dTAK1, Slpr, Mekk1, Ask1 and Wnd. All of them, except Wnd, have been previously implicated in cell death. ${ }^{16-21}$ In addition, dTAK1 plays a role in

\footnotetext{
${ }^{1}$ Institute of Intervention Vessel, Shanghai 10th People's Hospital, Shanghai Key Laboratory of Signaling and Disease Research, School of Life Science and Technology, Tongji University, Shanghai 200092, China

*Corresponding author: X Ma, Department of Molecular Biology and Genetics, Howard Hughes Medical Institute, Johns Hopkins University School of Medicine, Baltimore, MD 21205, USA. Tel: +1 443562 6219; Fax: +1 410502 3177; E-mail: XianjueMa@jhmi.edu

or L Xue, School of Life Science and Technology, Tongji University, 1239 Siping Road, Shanghai 200092, China. Tel: +86 13262557721; Fax: +86 21 65985407; E-mail: lei.xue@tongji.edu.cn

${ }^{2}$ Current address: Department of Molecular Biology and Genetics, Howard Hughes Medical Institute, Johns Hopkins University School of Medicine, Baltimore, MD 21205, USA.

Abbreviations: PCD, programmed cell death; JNK, c-Jun N-terminal kinase; A/P, anterior/posterior; $A O$, acridine orange

Received 17.10.14; revised 24.2.15; accepted 17.3.15; Edited by E Baehrecke
} 

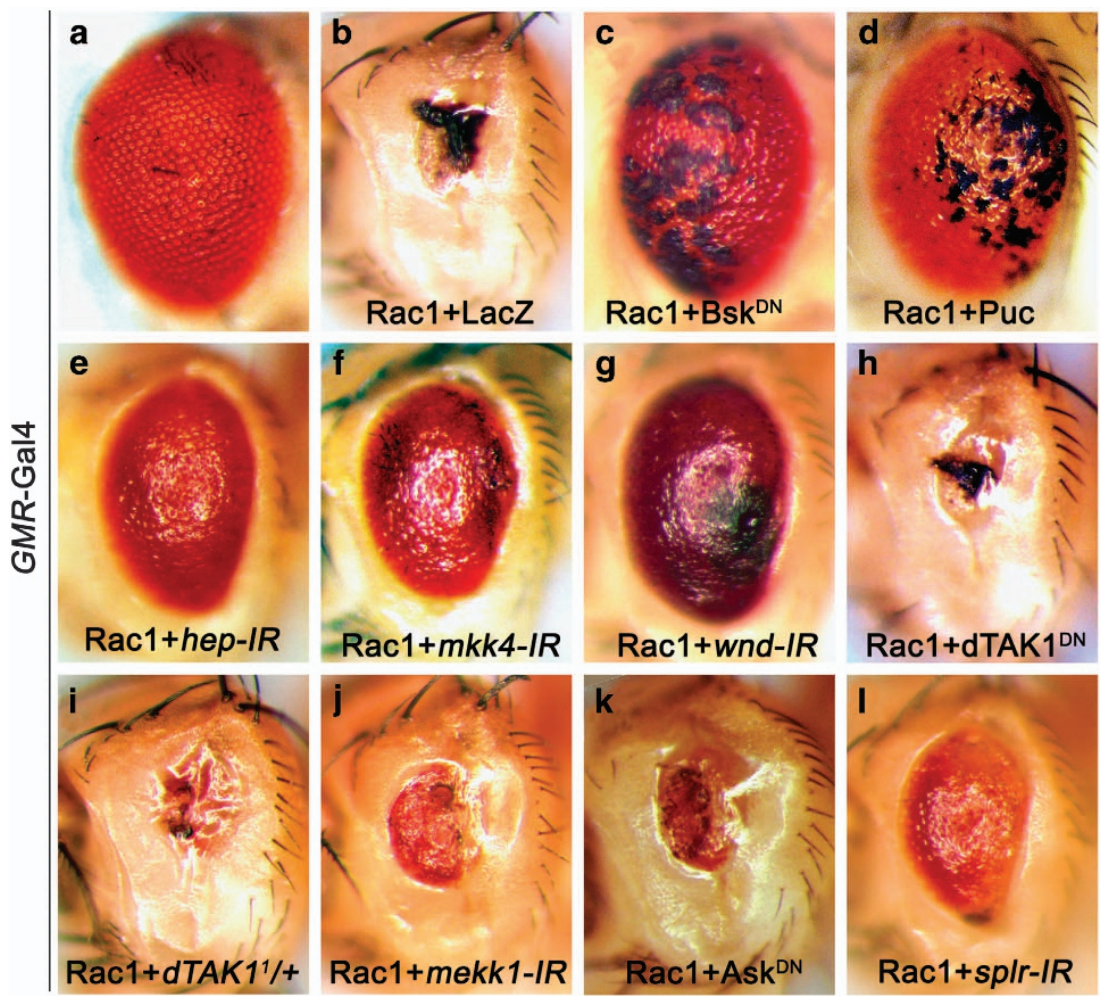

Rac1+wnd-IR
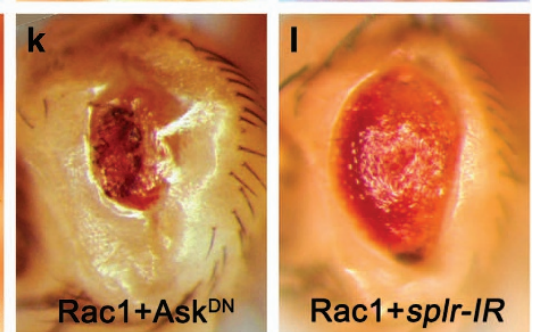

Figure 1 Wnd is essential for Rac1-induced small-eye phenotype. Light micrographs of Drosophila eyes are shown. Compared with the GMR-Gal4 control (a), GMR $>$ Rac1induced small-eye phenotype (b) was significantly suppressed by expression of Bsk ${ }^{\mathrm{DN}}$ (c) or Puc (d), or RNAi-mediated knocking down of hep (e), mkk4 (f) or wnd (g), and partially suppressed by knocking down mekk1 (j), Ask1 (k) or slpr (I), but remained unaffected by expression of dTAK1 ${ }^{\mathrm{DN}}(\mathrm{H})$ or mutation in dTAK1 (i)

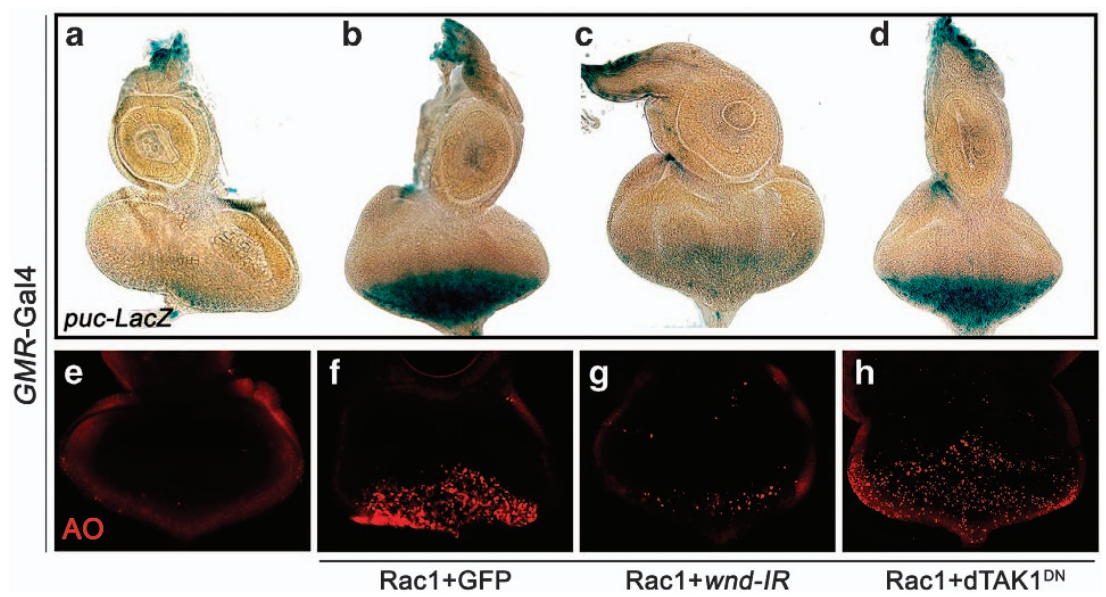

Figure 2 Wnd is required for Rac1-induced JNK activation and cell death. Light (a-d) and fluorescence (e-h) micrographs of Drosophila eye discs are shown. Compared with the GMR-Gal4 control (a and $\mathbf{e})$, Rac1-induced upregulated puc transcription (b) and cell death (f) posterior to MF in third instar eye discs were suppressed by the expression of a wnd RNAi (c and $\mathbf{g})$, but not that of $\operatorname{dTAK} 1^{\mathrm{DN}}(\mathbf{d}$ and $\mathbf{h})$

innate immunity, ${ }^{22}$ Slpr is required for dorsal closure, ${ }^{7}$ and Ask1 is involved in pigmentation. ${ }^{23}$ Wnd has been shown to play pivotal roles in regulating axon transportation, regeneration and degradation, ${ }^{24-26}$ but its role in cell death has remained unknown. We found $G M R>$ Rac1-induced no-eye phenotype was slightly suppressed by knocking down mekk1, Ask 1 or slpr (Figures $1 \mathrm{j}$ and I), but remained unaffected by expressing a dominant negative form of dTAK $1\left(\mathrm{dTAK} 1^{\mathrm{DN}}\right.$ ) or mutation in $d T A K 1$ (Figures $1 \mathrm{~h}$ and $\mathrm{i}$ ), suggesting dTAK1 is dispensable for Rac1-triggered cell death. Consistent with previous studies, expression of dTAK $1^{\mathrm{DN}}$ almost completely suppressed GMR $>$ Egr-induced small-eye phenotype (Supplementary Figures $1 \mathrm{~A}$ and $\mathrm{C}$ ), ${ }^{20,21,27,28}$ suggesting dTAK1 is specifically required for Egr- but not Rac1-triggered JNK-dependent cell death. Intriguingly, we found that knocking down wnd dramatically suppressed Rac1-induced no-eye phenotype (Figure 1g). Consistently, Rac1-induced JNK activation (indicated by puc-LacZ staining ${ }^{29}$ ) and cell death 
in developing eye disc were also suppressed by knocking down wnd (Figures $2 \mathrm{a}-\mathrm{c}$ and $\mathrm{e}-\mathrm{g}$ ), but remained unchanged by blocking dTAK1 activity (Figures $2 d$ and $h$ ). Together, the above data demonstrate that wnd plays a major role in mediating Rac1-triggered cell death in Drosophila.

Rac1-JNK signaling is also known to play essential role in the process of dorsal and thorax closure during normal development. ${ }^{10}$ In accordance with previous study, we found knocking down slpr in the thorax by pnr-Gal4 produced a cleft phenotype (Supplementary Figures 2A and B), ${ }^{7}$ whereas depletion of wnd produced no obvious phenotype (Supplementary Figure 2C). Thus, Wnd appears dispensable for the thorax closure function of Rac1-JNK signaling.

Wnd is physiologically required for JNK-mediated cell death. Correct establishment and maintenance of cell polarity are critical for development and tissue homeostasis. Loss of cell polarity results in JNK-dependent cell death and invasion in Drosophila. ${ }^{30-35}$ To investigate whether wnd is required for the physiological functions of JNK signaling in development, we knocked down scrib along the anterior/ posterior (A/P) compartment boundary in third instar larval wing discs by patched (ptc)-Gal4, and observed intensive cell death (indicated by cleaved Caspase 3 staining) and cell invasion into the posterior compartment (Figures 3b-b"). Depletion of wnd dramatically suppressed loss of scirbinduced cell death and invasion phenotypes (Figures 3c-c"), suggesting that Wnd also modulates the physiological functions of JNK signaling.
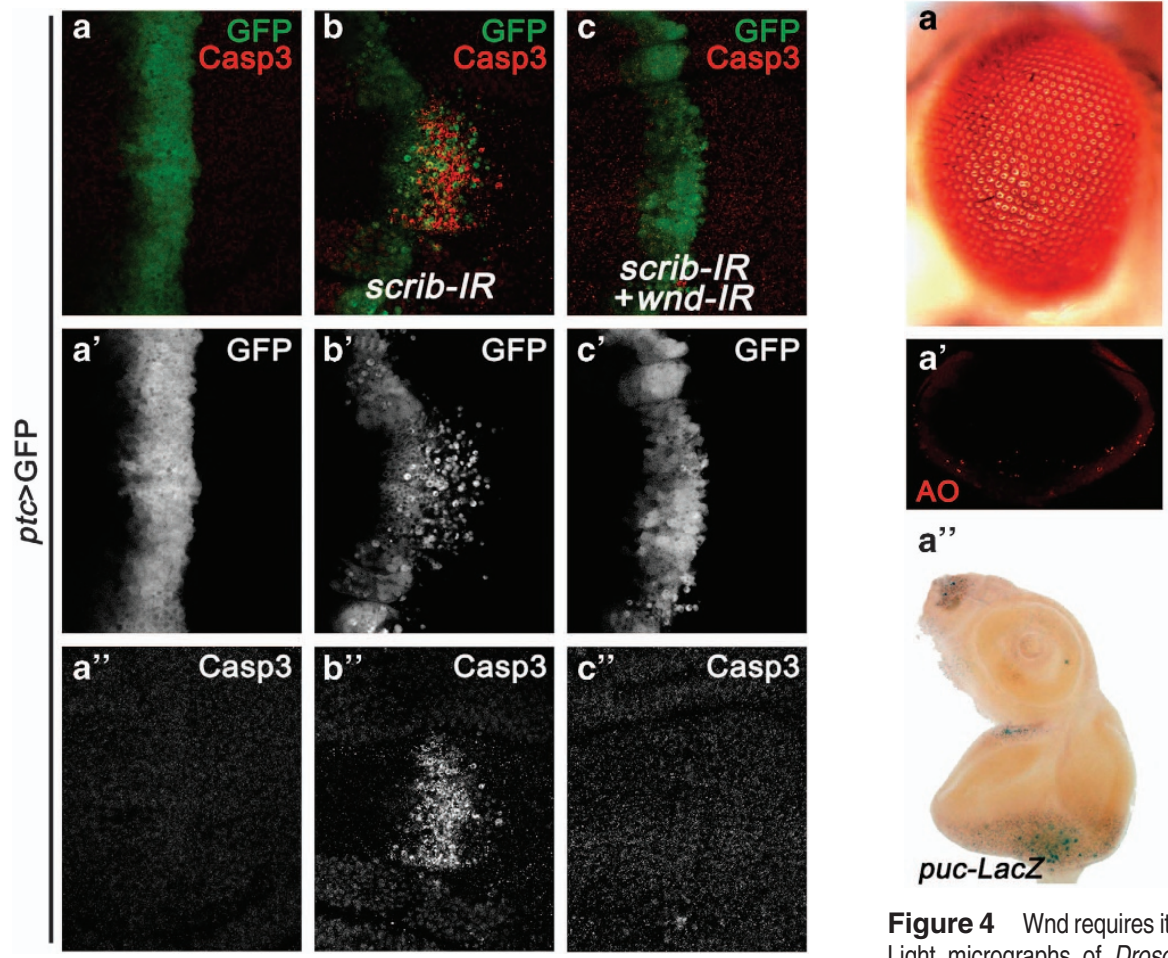

Figure 3 Wnd is required for loss of scrib-induced cell death. Fluorescence micrographs of Drosophila wing discs are shown. Compared with ptc-Gal4 control $(\mathbf{a}-\mathbf{a}$ "), loss of scrib-induced cell death and invasion (b-b") was strongly impeded by knocking down wnd (c-c")

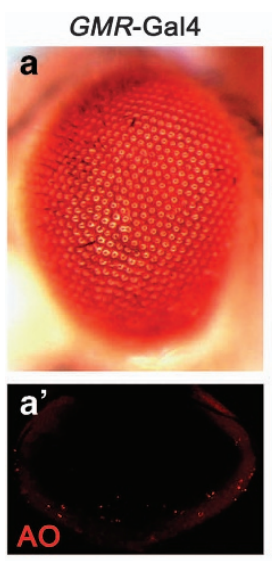

a"

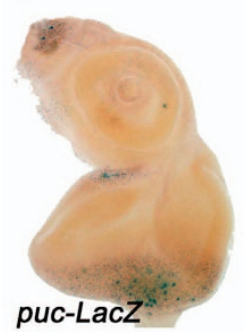

Wnd induces JNK-mediated cell death. Next, to examine whether Wnd is sufficient to induce JNK activation and cell death, we expressed Wnd in the developing eye under the GMR promoter, and observed a small-eye phenotype in the adults (Figure 4b). As expected, such phenotype could be suppressed by coexpression of a wnd RNAi (data not shown). In addition, Wnd prompts extensive cell death and JNK activation in third instar eye discs posterior to the MF, as indicated by $\mathrm{AO}$ staining (Figure 4b') and puc-LacZ expression (Figure 4b"), respectively. In contrast, a kinase-dead form of Wnd (Wnd ${ }^{\mathrm{KD}}$ ) ${ }^{25}$ fails to induce cell death and JNK activation in the eye disc, and produces a wild-type eye in the adults (Figures $4 \mathrm{c}-\mathrm{c}$ "), suggesting the kinase domain is necessary for Wnd to induce JNK activation and cell death. Finally, Wnd-triggered JNK activation, cell death and smalleye phenotype is fully suppressed by coexpression of Bsk ${ }^{\mathrm{DN}}$ or Puc (Figures 5g, g'), indicating Wnd triggers JNKdependent cell death.

MKK4 and Hep are both required for Wnd-induced cell death. Previous studies found DLK (Wnd ortholog in mammal) utilize MKK7 (Hep ortholog) but not MKK4 as a substrate in mammalian cells. ${ }^{36}$ To investigate whether Mkk4 or Hep is required for Wnd-induced JNK activation and cell death, we reduced their activities by mutations or RNAi expression. Intriguingly, loss of either hep or $m k k 4$ strongly blocked GMR $>$ Wnd-induced cell death in eye discs
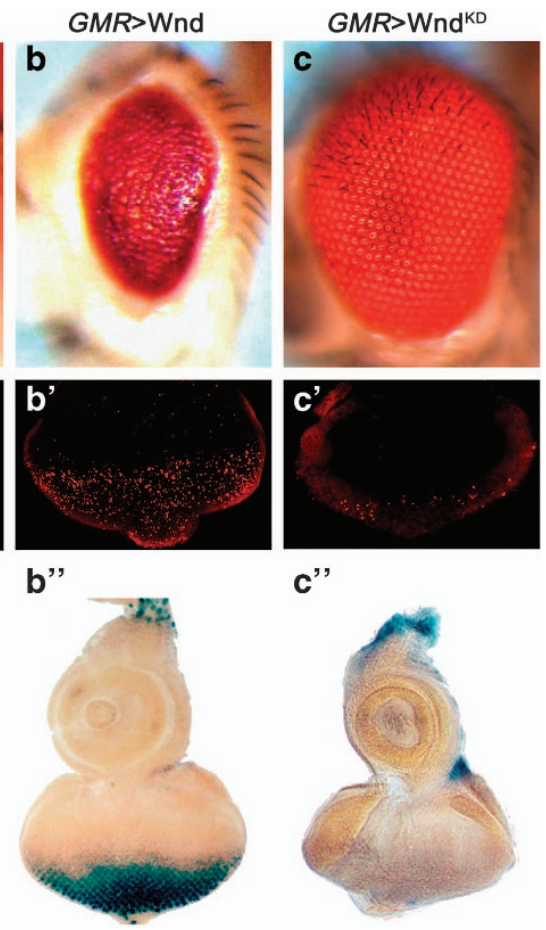

$c^{\prime \prime}$

Figure 4 Wnd requires its kinase domain to induce JNK activation and cell death. Light micrographs of Drosophila eyes (a-c), eye disc (a"-c") and fluorescence micrographs of eye discs (a'-c') are shown. Compared with the control (a-a"), expression of Wnd induced extensive cell death ( $\left.\mathbf{b}^{\prime}\right)$ and elevated puc transcription (b") in eye discs, and produced a small-eye phenotype in adults (b), whereas expression of Wnd ${ }^{\mathrm{KD}}$ produced no obvious phenotypes (c-c") 

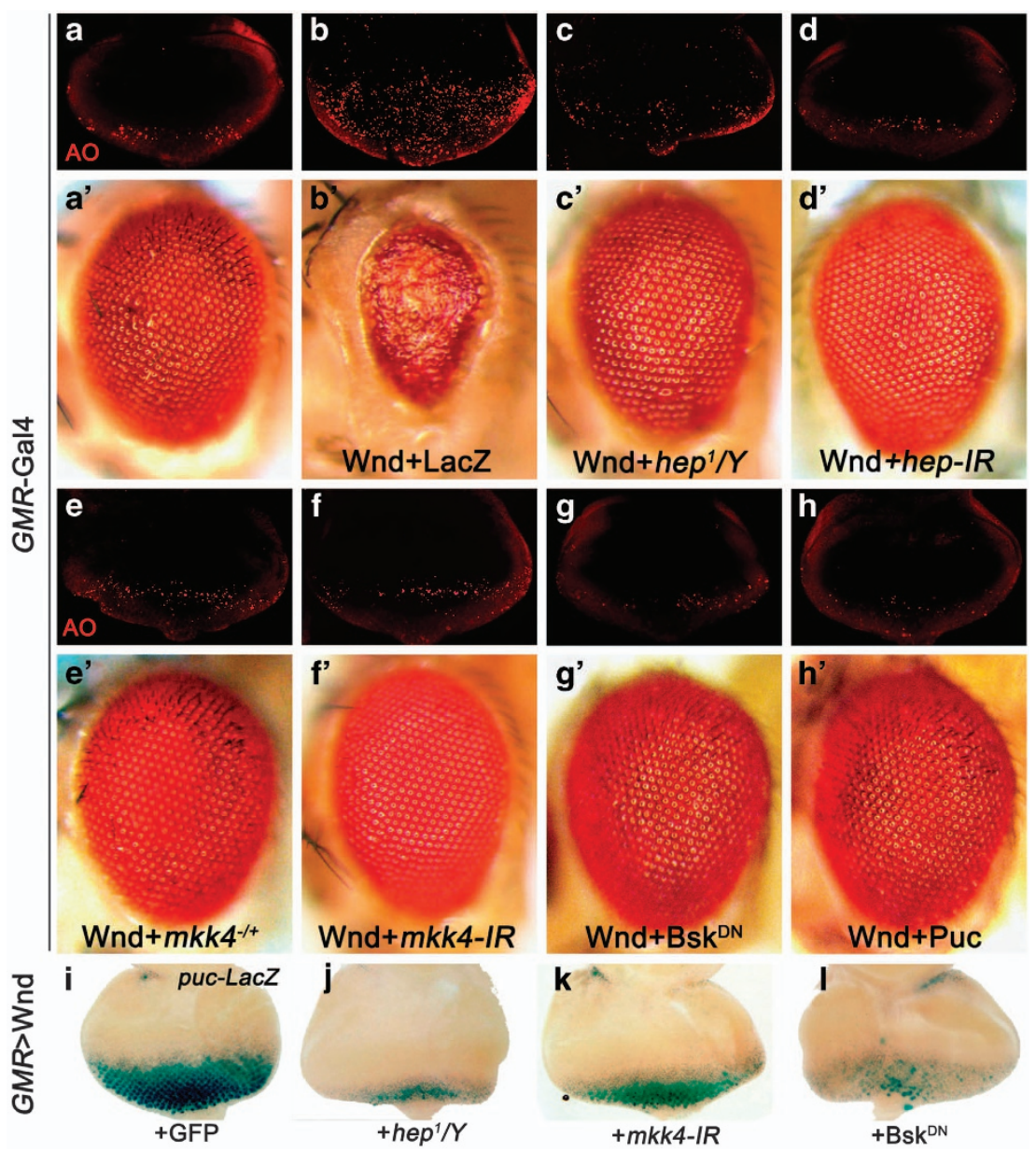

Figure 5 Wnd acts through MKK4 and Hep to induce JNK-dependent cell death. (a-h) Fluorescence micrographs of Drosophila eye discs (a-h) and light micrographs of adult eyes $\left(\mathbf{a}^{\prime}-\mathbf{h}^{\prime}\right)$ are shown. Compared with the control (a), Wnd-induced cell death and small-eye phenotype (b) could be strongly suppressed by loss of hep (c and $\mathbf{d}$ ) or $m k k 4$ (e and f), or expression of Bsk ${ }^{\mathrm{DN}}(\mathbf{g})$ or Puc (h). (i-l) Light micrographs of Drosophila eye disc are shown. Wnd-induced puc-LacZ expression (i) was impeded by loss of hep (j) or mkk4 (k), or expression of Bsk ${ }^{\mathrm{DN}}(\mathrm{I})$

(Figures $5 c-f$ ) and the small-eye phenotype in adults (Figures $\left.5 c^{\prime}-f^{\prime}\right)$, suggesting both MKK4 and Hep are necessary for Wnd-induced cell death in vivo. Consistently, both MKK4 and Hep are required for Wnd-triggered JNK activation, as loss of either gene strongly suppressed Wnd-induced puc-LacZ expression (Figures $5 \mathrm{i}-\mathrm{k}$ ). Collectively, these results imply that MKK4 and Hep might work together rather than in parallel, for instance in the same complex, to mediate Wndtriggered JNK activation and cell death in Drosophila.

Wnd is required for Egr-induced cell death and JNK activation. It has been reported that both MKK4 and Hep are required for cell death induced by Egr (Figures 6b, e and f), the Drosophila ortholog of TNF. ${ }^{37}$ Although dTAK1 has been previously implicated in Egr-induced JNK activation and cell death, ${ }^{20,38}$ a potential role of Wnd in Egr-JNK signaling cannot be excluded. Indeed, we found that knocking down wnd partially suppressed $G M R>$ Egr-induced cell death and JNK activation in eye discs (Figures 6a'-c' and a"-c"), and the small-eye phenotype in adults (Figures $6 a-c$ ). This suppression was further confirmed in wnd mutants (Figure 6d), suggesting Wnd also contributes to Egr-induced cell death. However, inactivation of dTAK1 almost fully blocked $G M R>$ Egr-induced small-eye phenotype (Supplementary Figure 1C), suggesting dTAK1 is the major MAPKKK in Egr-induced JNK-dependent cell death. Furthermore, in accordance with the role of Rac1 in regulating JNKmediated cell death, we found Rac1 is also required for GMR $>$ Egr-induced small eye and cell death (Supplementary Figures $1 \mathrm{~B}$ and $\mathrm{E})$.

To investigate whether Wnd is required for Egr-induced cell death in a nontissue-specific manner, we characterized the genetic interaction between Wnd and Egr in the developing wing. Expression of Egr driven by ptc-Gal4 triggers cell death in the wing disc and generates a loss of anterior crossvein (acv) phenotype in the adult wing, both of which were strongly suppressed by loss of wnd (Figures 6g-l, g'-i' and s). Furthermore, ptc $>$ Egr-induced puc-LacZ expression in the wing disc was also suppressed by depletion of wnd (Figures 6m-o). Together, these results demonstrate that Wnd is required for Egr-induced JNK activation and cell death in wing development.

Furthermore, expression of Wnd driven by ptc-Gal4 recapitulates the loss-of-acv phenotype of ptc $>$ Egr 

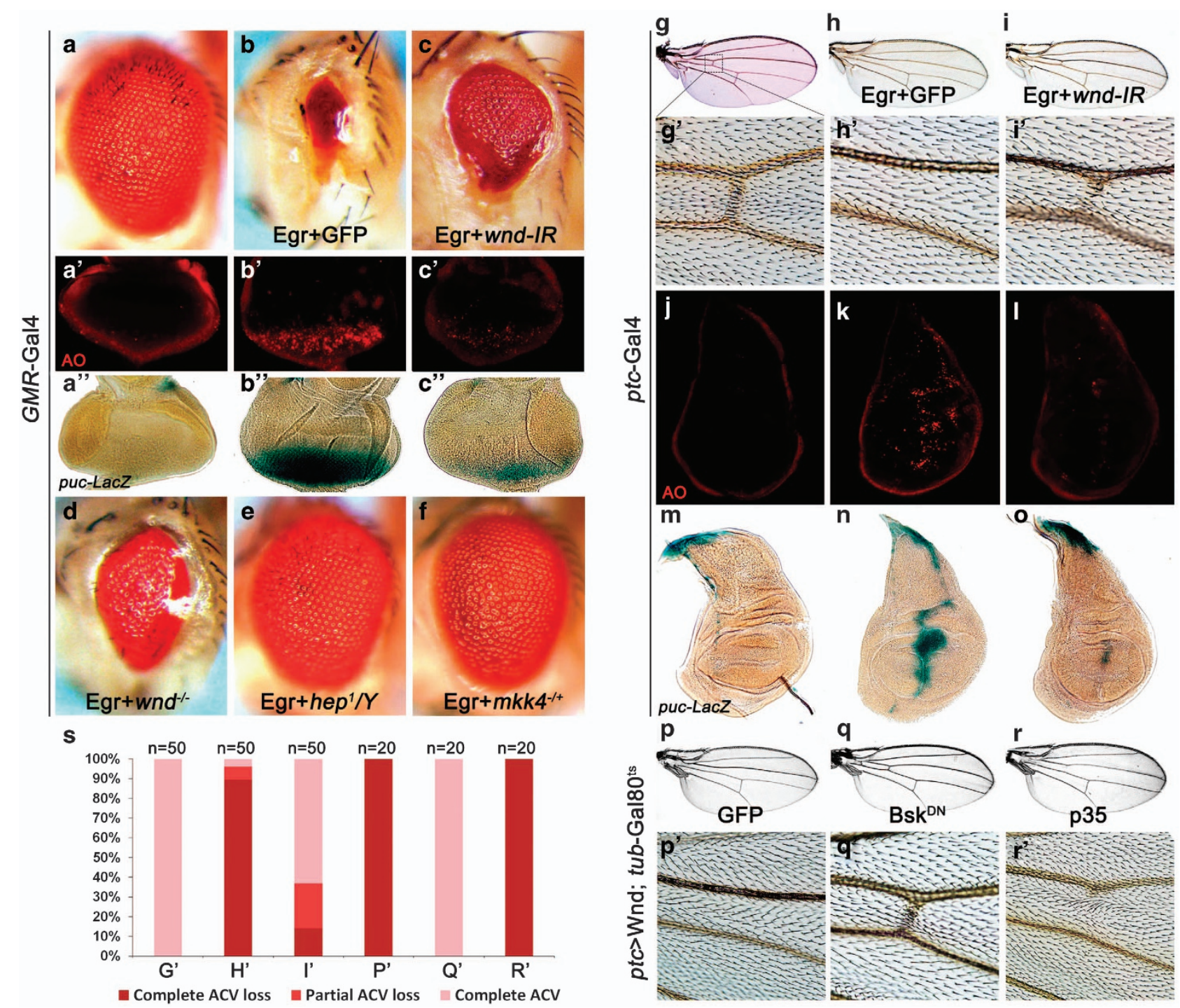

Figure 6 Wnd regulates Egr-induced cell death and JNK activation. (a-f) Compared with the GMR-Gal4 control (a-a"), Egr-induced small-eye phenotype (b), cell death (b') and puc-LacZ expression (b") were suppressed partially by knocking down wnd (c-c"). The GMR $>$ Egr small-eye phenotype was suppressed partially in wnd mutants $\left(\mathbf{d}, w_{n} d^{1} / w n d^{3}\right)$, but near fully in hemizygous hep (e, hep ${ }^{1} M$ or heterozygous $m k k 4\left(\mathbf{f}, m k k 4^{G 673} /+\right)$ mutants. $(\mathbf{g}-\mathbf{0})$ Compared with the ptc-Gal4 control (g, j jand $\left.\mathbf{m}\right)$, Egr-triggered cell death $(\mathbf{k})$ and JNK activation $(\mathbf{n})$ in wing discs and the loss of anterior crossvein in adult wings ( $\mathbf{h}$ and $\mathbf{h}$ ') were suppressed by knocking down wnd (i, $\mathbf{i}^{\prime}, \mathbf{I}$ and $\left.\mathbf{o}\right)$. ( $\left.\mathbf{p}-\mathbf{r}\right)$ Wndinduced loss of anterior crossvein phenotype ( $\mathbf{p}$ and $\mathbf{p}^{\prime}$ ) was completely suppressed by the expression of Bsk ${ }^{\mathrm{DN}}$ ( $\mathbf{q}$ and $\left.\mathbf{q}^{\prime}\right)$, but not that of p35 ( $\mathbf{r}$ and $\left.\mathbf{r}^{\prime}\right)$. ( $\left.\mathbf{s}\right)$ Quantification data of loss of anterior crossvein phenotype in ( $\mathbf{g}^{\prime}, \mathbf{h}^{\prime}, \mathbf{l}^{\prime}, \mathbf{p}^{\prime}, \mathbf{q}^{\prime}$ and $\left.\mathbf{r}^{\prime}\right)$

(Figures 6p, p' and s). As ptc $>$ Wnd results in lethality at larva stage, we used tub-Gal80 ${ }^{\text {ts }}$ expressing a temperaturesensitive form of the Gal4 inhibitor Gal80 (Gal80 ${ }^{\text {ts }}$ ) to block Gal4 activity at low temperature $\left(18^{\circ} \mathrm{C}\right)$, and to unchain the inhibition at high temperature $\left(29^{\circ} \mathrm{C}\right) .^{39}$ Interestingly, ptc $>$ Wnd-induced loss-of-acv phenotype was fully suppressed by inactivation of JNK (Figures 6q, q' and s), but remained unaffected by expression of p35 that blocks caspase's activity (Figures 6r, r' and s). These data are consistent with our previous report that JNK signaling induces caspase-independent cell death. ${ }^{27}$

Wnd acts in parallel with dTAK1 in the TNF-JNK signaling pathway. The above results suggest that Wnd may act as a novel component in the TNF-JNK signaling pathway. To further genetically map Wnd in this pathway, we performed epistasis analysis between Wnd and dTAK1 or Hep. Consistent with previous data, expression of a constitutive activated form of Hep $\left(\mathrm{Hep}^{\mathrm{CA}}\right)$ in the developing eye under GMR promoter induced JNK-mediated cell death and resulted in a small-eye phenotype (Figure 7a). ${ }^{27,32}$ This phenotype could not be suppressed by loss of Wnd (Figure 7b), consistent with our genetic data that Hep is required for Wnd-induced cell death (Figures $5 \mathrm{c}$ and $\mathrm{d}$ ). Furthermore, we found dTAK1-triggered rough-eye phenotype (Figure $7 d)^{34}$ remained unaffected by the loss of Wnd (Figure 7e). Conversely, blocking dTAK1 activity could not suppress Wnd-induced small-eye phenotype as well (Figures $7 \mathrm{~g}$ and $\mathrm{h}$ ). As a positive control, the eye phenotype induced by ectopic expression of $\mathrm{Hep}^{\mathrm{CA}}$, dTAK1 or Wnd was 

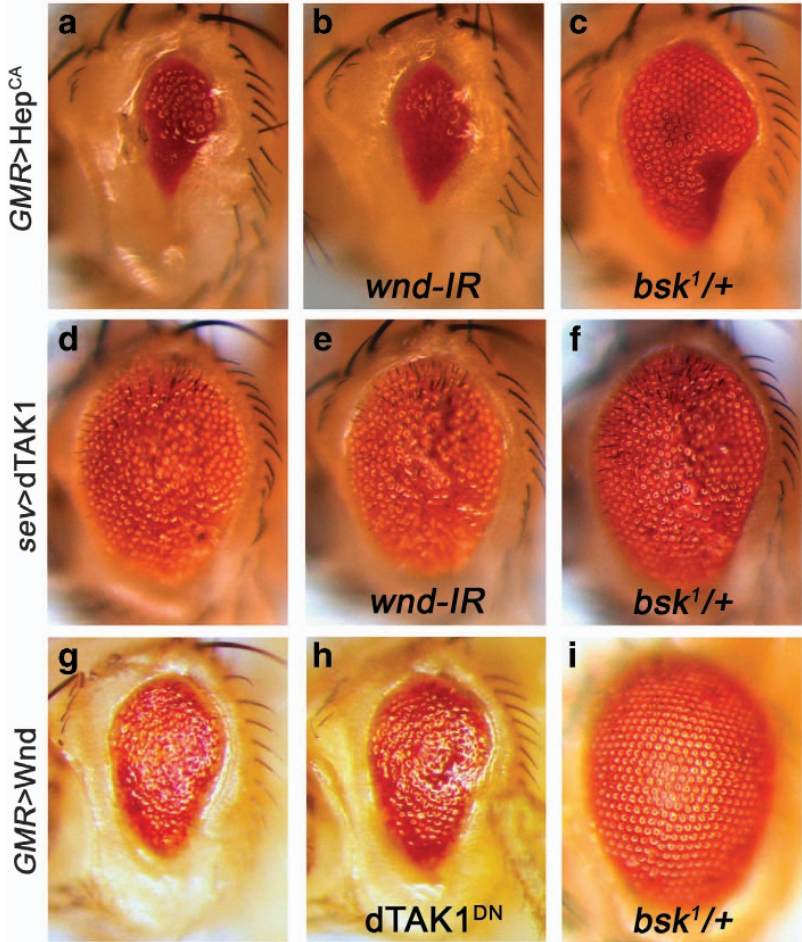

Figure 7 Wnd acts in parallel with dTAK1 in Egr-JNK pathway. Light micrographs of Drosophila eyes are shown. The small-eye phenotype of GMR $>\mathrm{Hep}^{\mathrm{CA}}$ (a) and the rough eye of sev $>$ dTAK1 (d) was not suppressed by knocking down wnd (b and $\mathbf{e}$ ), but was significantly suppressed in heterozygous bsk mutants (c and f). Conversely, GMR $>$ Wnd-induced small-eye phenotype $(\mathbf{g})$ was not affected by blocking dTAK1 activity (h), but was dramatically suppressed in heterozygous bsk mutants (i)

significantly suppressed by a mutation in one copy of endogenous bsk (Figures 7c, f and i; Supplementary Figure 3). Together, these results indicate that Wnd acts in parallel with dTAK1 in regulating JNK-mediated cell death.

Wnd is required for Rho1-induced cell death. Apart from Rac1, another Rho GTPase family member Rho1 has been implicated in cell death and neurodegeneration. ${ }^{18,40}$ In accordance with these findings, we found ectopic Rho1 expression driven by GMR-Gal4 resulted in increased cell death and JNK activation in third instar eye discs and produced a small rough-eye phenotype in adults (Figures 8b-b"). These phenotypes were suppressed by knocking down wnd (Figures 8c-c"), suggesting Wnd is also required for Rho1induced JNK activation and cell death. Intriguingly, loss of wnd fully suppressed Rho1-induced JNK activation, cell death and reduced eye size, but not the rough-eye phenotype (Figures $8 c-c$ "), suggesting Rho1-induced eye roughness is likely independent of JNK signaling. Consistent with this explanation, blocking JNK activity by knocking down hep or $m k k 4$, or expressing Puc, was able to suppress the reduced size, but not the roughness, of $G M R>$ Rho1 adult eyes (Figures 8d-f).

\section{Materials and Methods}

Drosophila stocks and genetics. All stocks were raised on standard Drosophila media and crosses were performed at $25^{\circ} \mathrm{C}$ unless otherwise indicated.

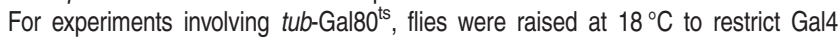

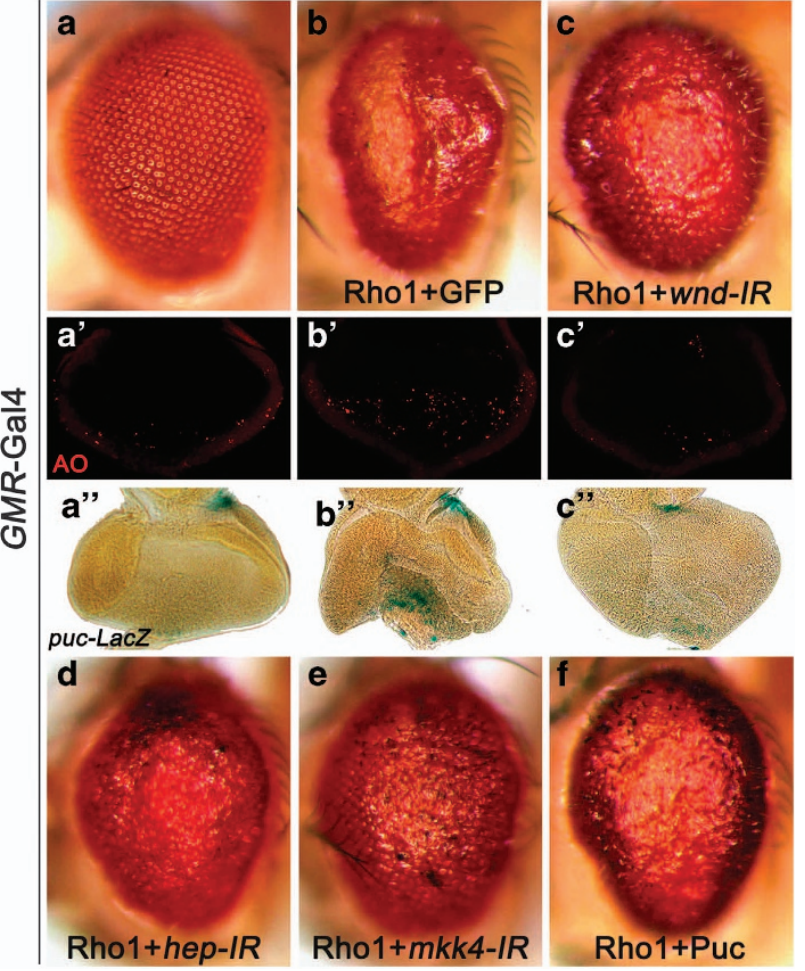

Figure 8 Wnd is required for Rho1-induced cell death and JNK activation. Compared with the control (a-a"), GMR $>$ Rho1-triggered cell death (b') and pucLacZ expression (b") in eye discs and small-eye phenotype (b) were significantly suppressed by knocking down wnd (c-c"). The GMR $>$ Rho1 small-eye phenotype was also suppressed by knocking down hep (d) or mkk4 (e), or expression of Puc (f)

activity for 5-6 days, then shifted to $29^{\circ} \mathrm{C}$ for 2 days to inactivate Gal $80^{\text {ts }}$. The following stocks were used: GMR-Gal4, ptc-Gal4, sev-Gal4, UAS-GFP, UAS-Rac1 (6680), UAS-Rho1 (7334), UAS-LacZ (3956) and wnd ${ }^{\text {Exel6135 }}$ (7614, EP line use for overexpression), all obtained from the Bloomington Stock Center (Bloomington, IN, USA), UAS-Rac1-IR (2248R-1) ${ }^{43}$ obtained from National Institute of Genetics (NIG, Mishima, Japan), UAS-Wnd ${ }^{\mathrm{KD}}$, wnd ${ }^{1}$, wnd ${ }^{3}$ (gifts from Aaron DiAntonio, St. Louis, MO, USA), UAS-Ask1 ${ }^{\text {DN }}$ (gift from Masayuki Miura, Tokyo, Japan), hep $^{1}$, UAS-Egr, UAS-dTAK1, UAS-dTAK1 ${ }^{\mathrm{DN}}$, UAS-Bsk ${ }^{\mathrm{DN}}$, UAS-hep-IR, UAS-Puc, puc $^{E 69} 44$ bsk $^{134}$ UAS-Hep ${ }^{\text {CA }}$, dTAK1 $1^{127}$ UAS-wnd-IR, ${ }^{24}$ UAS-MKK4-IR, ${ }^{43}$ $m k k 4^{G 673,37} U A S-s / p r-I R^{18}$ and UAS-mekk1-IR, ${ }^{45}$ as previously described.

Immunostaining. Third instar larvae wing discs were fixed in freshly made $4 \%$ paraformaldehyde for 15 min and washed 3 times with $1 \times$ PBS, then stained using rabbit anti-active Caspase $3(1: 200)$ (Cell Signaling Technology, Danvers, MA, USA). Secondary antibody was anti-rabbit-Cy3 (1:1000, Jackson Immunochemicals, West Grove, PA, USA).

X-gal staining. Eye and wing discs were dissected from third instar larvae in PBST $(1 \times$ PBS pH 7.0, 0.1\% Triton X-100) and stained for $\beta$-galactosidase activity.

AO staining. AO staining was done as previously described. ${ }^{33}$ Briefly, eye or wing discs were dissected from late third instar larvae in PBST and incubated in $1 \times 10^{-5} \mathrm{M} \mathrm{AO}$ for $5 \mathrm{~min}$ at room temperature before imaging.

\section{Conclusions}

We have uncovered Wnd as a crucial regulator of JNK-mediated cell death in Drosophila. Specifically, our genetic epistasis analysis established Wnd as a novel cell death modulator downstream of Rac1, Egr, Rho1 and loss of scrib. Furthermore, we show that Wnd is sufficient to induce JNK-dependent cell death through both MKK4 and Hep, and this is different from previous mammalian study that DLK utilize only MKK7 as its substrate. ${ }^{36}$ Our finding also clarifies the independent roles of Wnd 
and the well-known JNKKK dTAK1 in regulating JNK-mediated cell death. Whereas dTAK1 is required only for Egr- but not Rac1-triggered cell death, Wnd plays crucial roles in both situations. Furthermore, Wnd and dTAK1 act in parallel to regulate JNKdependent cell death upstream of MKK4 and Hep. Besides the established role in cell death, the Drosophila JNK pathway is also required for cell migration and tumor metastasis. ${ }^{2,34,41}$ Consistent with this notion, loss of DLK, the mammalian ortholog of Wnd, results in delayed radial migration of neuronal cells. ${ }^{42}$ Therefore, a potential role of Wnd in regulating cell migration and tumor metastasis is worth further investigation.

\section{Conflict of Interest}

The authors declare no conflict of interest.

Acknowledgements. We thank Aaron DiAntonio, Konrad Basler, Bloomington, VDRC and NIG stock centers for fly stocks and reagents. This research was supported by the National Basic Research Program of China (973 Program) (2011CB943903), National Natural Science Foundation of China (31071294, 31171413 and 31371490 ), the PhD Programs Foundation of Ministry of Education of China $(20120072110023,20120072120030)$ and Shanghai Committee of Science and Technology (09DZ2260100, 14JC1406000).

1. Fuchs Y, Steller H. Programmed cell death in animal development and disease. Cell 2011; 147: 742-758.

2. Pastor-Pareja JC, Xu T. Dissecting social cell biology and tumors using Drosophila genetics. Annu Rev Genet 2013; 47: 51-74.

3. Adams MD, Celniker SE, Holt RA, Evans CA, Gocayne JD, Amanatides PG et al. The genome sequence of Drosophila melanogaster. Science 2000; 287: 2185-2195.

4. Richardson H, Kumar S. Death to flies: Drosophila as a model system to study programmed cell death. J Immunol Methods 2002; 265: 21-38.

5. Varfolomeev EE, Ashkenazi A. Tumor necrosis factor: an apoptosis JuNKie? Cell 2004; 116 491-497.

6. Stefanatos RK, Vidal M. Tumor invasion and metastasis in Drosophila: a bold past, a bright future. J Genet Genomics 2011; 38: 431-438.

7. Stronach B, Perrimon N. Activation of the JNK pathway during dorsal closure in Drosophila requires the mixed lineage kinase, slipper. Genes Dev 2002; 16: 377-387.

8. Williams MJ, Wiklund ML, Wikman S, Hultmark D. Rac1 signalling in the Drosophila larval cellular immune response. J Cell Sci 2006; 119: 2015-2024.

9. Baek SH, Kwon YC, Lee H, Choe KM. Rho-family small GTPases are required for cell polarization and directional sensing in Drosophila wound healing. Biochem Biophys Res Commun 2010; 394: 488-492.

10. Woolner S, Jacinto A, Martin P. The small GTPase Rac plays multiple roles in epithelial sheet fusion-dynamic studies of Drosophila dorsal closure. Dev Biol 2005; 282: 163-173.

11. Geisbrecht ER, Montell DJ. A role for Drosophila IAP1-mediated caspase inhibition in Rac-dependent cell migration. Cell 2004; 118: 111-125.

12. Lee NK, Choi HK, Kim DK, Lee SY. Rac1 GTPase regulates osteoclast differentiation through TRANCE-induced NF-kappa B activation. Mol Cell Biochem 2006; 281: 55-61.

13. Higuchi $Y$, Otsu K, Nishida K, Hirotani S, Nakayama H, Yamaguchi $O$ et al. The small GTPbinding protein Rac1 induces cardiac myocyte hypertrophy through the activation of apoptosis signal-regulating kinase 1 and nuclear factor-kappa B. J Biol Chem 2003; 278: 20770-20777

14. Hu H, Li M, Labrador JP, McEwen J, Lai EC, Goodman CS et al. Cross GTPase-activating protein (CrossGAP)/Vilse links the Roundabout receptor to Rac to regulate midline repulsion. Proc Natl Acad Sci USA 2005; 102: 4613-4618.

15. Abrams JM, White K, Fessler LI, Steller H. Programmed cell death during Drosophila embryogenesis. Development 1993; 117: 29-43.

16. Kang MJ, Chung J, Ryoo HD. CDK5 and MEKK1 mediate pro-apoptotic signalling following endoplasmic reticulum stress in an autosomal dominant retinitis pigmentosa model. Nat Cell Biol 2012; 14: 409-415

17. Takatsu Y, Nakamura M, Stapleton M, Danos MC, Matsumoto K, O'Connor MB et al. TAK1 participates in c-Jun $\mathrm{N}$-terminal kinase signaling during Drosophila development. Mol Cell Biol 2000; 20: 3015-3026.

18. Neisch AL, Speck O, Stronach B, Fehon RG. Rho1 regulates apoptosis via activation of the JNK signaling pathway at the plasma membrane. J Cell Biol 2010; 189: 311-323.

19. Ryabinina OP, Subbian E, Iordanov MS. D-MEKK1, the Drosophila orthologue of mammalian MEKK4/MTK1, and Hemipterous/D-MKK7 mediate the activation of D-JNK by cadmium and arsenite in Schneider cells. BMC Cell Biol 2006; 7: 7.

20. Igaki T, Kanda H, Yamamoto-Goto Y, Kanuka H, Kuranaga E, Aigaki T et al. Eiger, a TNF superfamily ligand that triggers the Drosophila JNK pathway. EMBO J 2002; 21: 3009-3018.
21. Geuking P, Narasimamurthy R, Basler K. A genetic screen targeting the tumor necrosis factor/Eiger signaling pathway: identification of Drosophila TAB2 as a functionally conserved component. Genetics 2005; 171: 1683-1694.

22. Park JM, Brady H, Ruocco MG, Sun H, Williams D, Lee SJ et al. Targeting of TAK1 by the NF-kappa B protein Relish regulates the JNK-mediated immune response in Drosophila. Genes Dev 2004; 18: 584-594.

23. Sekine Y, Takagahara S, Hatanaka R, Watanabe T, Oguchi H, Noguchi T et al. p38 MAPKs regulate the expression of genes in the dopamine synthesis pathway through phosphorylation of NR4A nuclear receptors. J Cell Sci 2011; 124: 3006-3016.

24. Xiong $X$, Collins CA. A conditioning lesion protects axons from degeneration via the Wallenda/DLK MAP kinase signaling cascade. J Neurosci 2012; 32: 610-615.

25. Collins CA, Wairkar YP, Johnson SL, DiAntonio A. Highwire restrains synaptic growth by attenuating a MAP kinase signal. Neuron 2006; 51: 57-69.

26. Hammarlund M, Nix P, Hauth L, Jorgensen EM, Bastiani M. Axon regeneration requires a conserved MAP kinase pathway. Science 2009; 323: 802-806.

27. Ma X, Huang J, Yang L, Yang Y, Li W, Xue L. NOPO modulates Egr-induced JNKindependent cell death in Drosophila. Cell Res 2012; 22: 425-431.

28. Kanda H, Miura M. Regulatory roles of JNK in programmed cell death. J Biochem 2004; 136 : 1-6.

29. Agnes F, Suzanne M, Noselli S. The Drosophila JNK pathway controls the morphogenesis of imaginal discs during metamorphosis. Development 1999; 126: 5453-5462.

30. Brumby AM, Richardson HE. scribble mutants cooperate with oncogenic Ras or Notch to cause neoplastic overgrowth in Drosophila. EMBO J 2003; 22: 5769-5779.

31. Igaki T, Pastor-Pareja JC, Aonuma H, Miura M, Xu T. Intrinsic tumor suppression and epithelial maintenance by endocytic activation of Eiger/TNF signaling in Drosophila. Dev Cell 2009; 16: 458-465

32. Ma X, Yang L, Yang Y, Li M, Li W, Xue L. dUev1a modulates TNF-JNK mediated tumor progression and cell death in Drosophila. Dev Biol 2013; 380: 211-221.

33. Ma X, Shao Y, Zheng H, Li M, Li W, Xue L. Src42A modulates tumor invasion and cell death via Ben/dUev1a-mediated JNK activation in Drosophila. Cell Death Dis 2013; 4: e864.

34. Ma X, Li W, Yu H, Yang Y, Li M, Xue L et al. Bendless modulates JNK-mediated cell death and migration in Drosophila. Cell Death Differ 2014; 21: 407-415.

35. Ma X. Context-dependent interplay between Hippo and JNK pathway in Drosophila. AIMS Genet 2014; 1: 20-33.

36. Merritt SE, Mata M, Nihalani D, Zhu C, Hu X, Holzman LB. The mixed lineage kinase DLK utilizes MKK7 and not MKK4 as substrate. J Biol Chem 1999; 274: 10195-10202.

37. Geuking P, Narasimamurthy R, Lemaitre B, Basler K, Leulier F. A non-redundant role for Drosophila Mkk4 and hemipterous/Mkk7 in TAK1-mediated activation of JNK. PLoS One 2009; 4: e7709.

38. Moreno E, Yan M, Basler K. Evolution of TNF signaling mechanisms: JNK-dependent apoptosis triggered by Eiger, the Drosophila homolog of the TNF superfamily. Curr Biol 2002; 12: $1263-1268$

39. McGuire SE, Le PT, Osborn AJ, Matsumoto K, Davis RL. Spatiotemporal rescue of memory dysfunction in Drosophila. Science 2003; 302: 1765-1768.

40. Cook M, Mani P, Wentzell JS, Kretzschmar D. Increased RhoA prenylation in the loechrig (loe) mutant leads to progressive neurodegeneration. PLoS One 2012; 7: e44440.

41. Igaki T, Pagliarini RA, Xu T. Loss of cell polarity drives tumor growth and invasion through JNK activation in Drosophila. Curr Biol 2006; 16: 1139-1146.

42. Hirai S, Cui de F, Miyata T, Ogawa M, Kiyonari H, Suda Y et al. The c-Jun N-terminal kinase activator dual leucine zipper kinase regulates axon growth and neuronal migration in the developing cerebral cortex. J Neurosci 2006; 26: 11992-12002.

43. Lesch C, Jo J, Wu Y, Fish GS, Galko MJ. A targeted UAS-RNAi screen in Drosophila larvae identifies wound closure genes regulating distinct cellular processes. Genetics 2010; 186: 943-957.

44. Xue L, Igaki T, Kuranaga E, Kanda H, Miura M, Xu T. Tumor suppressor CYLD regulates JNK-induced cell death in Drosophila. Dev Cell 2007; 13: 446-454.

45. Brun S, Vidal S, Spellman P, Takahashi K, Tricoire H, Lemaitre B. The MAPKKK Mekk1 regulates the expression of Turandot stress genes in response to septic injury in Drosophila. Genes Cells 2006; 11: 397-407.

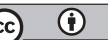

Cell Death and Disease is an open-access journal published by Nature Publishing Group. This work is licensed under a Creative Commons Attribution 4.0 International License. The images or other third party material in this article are included in the article's Creative Commons license, unless indicated otherwise in the credit line; if the material is not included under the Creative Commons license, users will need to obtain permission from the license holder to reproduce the material. To view a copy of this license, visit http://creativecommons.org/licenses/by/4.0/ 